\title{
Regiōes Urbanas Homogêneas no Brasil: Uma Análise Espacial Aplicada à Distribuição Populacional'
}

\section{Functional Urban Regions in Brazil: A Spatial Analysis Applied to Population Distribution}

\author{
Tarcha, Luciana lannone1; Manzato, Gustavo Garcia² \\ ' Universidade Estadual Paulista (UNESP), Campus Bauru, Bauru, Brasil; \\ luciana.tarcha@gmail.com \\ 2 Universidade Estadual Paulista (UNESP), Campus Bauru, Bauru, Brasil; \\ gustavo.manzato@unesp.br
}

\begin{abstract}
RESUMO
Diversas Regiões Urbanas Homogêneas (RUHs) estabelecidas no país possuem uma fraca ou praticamente nenhuma dinâmica metropolitana e foram criadas segundo critérios políticoadministrativos. Existe, portanto, uma necessidade de criar modelos que possam auxiliar na definição das RUHs. O objetivo deste trabalho de mestrado foi identificar as RUHs no Brasil por meio da estatística espacial, utilizando dados da distribuição populacional. Aqui serão apresentados os resultados iniciais deste projeto, no qual foi realizada uma análise das RUHs considerando-se todo o território nacional por meio da utilização de ferramentas de estatística espacial aplicadas à densidade populacional, uma vez que tal análise ainda não havia sido desenvolvida. O estudo pôde identificar as RUHs brasileiras segundo um critério mais quantitativo, apontando regiões em que a instalação de governos metropolitanos foi feita sem critério padronizado ou quantitativo, considerando que as RUHs apontadas pelo método aqui empregado não coincidem inteiramente com os limites das RUHs oficiais.
\end{abstract}

Palavras-chave: Regiões Urbanas Homogêneas, Análise Espacial, Densidade Populacional.

\begin{abstract}
Many Functional Urban Regions (FURs) established in the country show a weak, or even a lack of metropolitan dynamics and were created according to political-administrative criteria. Therefore, there is a necessity for creating models that could support the definition of FURs. The main objective of this Master's work is to identify the FURs in Brazil through spatial statistics, using the population distribution data. The initial results of this project will be presented here, in which a FURs analysis was made considering all national territory by using spatial statistics tools applied to population density, since this analysis had not been made yet. This study was able to identify Brazilian FURs according to a more quantitative criterion, identifying regions where the installation of a metropolitan government was made without a standardized or
\end{abstract}

\footnotetext{
${ }^{1}$ TARCHA, Luciana lannone; MANZATO, Gustavo Garcia. Regiões Urbanas Homogêneas no Brasil: Uma Análise Espacial Aplicada à Distribuição Populacional. In: II SIMPÓSIO NACIONAL DE GESTÃO E ENGENHARIA URBANA: SINGEURB, 2019, São Paulo. Anais... Porto Alegre: ANTAC, 2019.
} 
quantitative criterion, whereas the FURs detected by the applied method did not match entirely with the limits of the official FURs.

Keywords: Functional Urban Regions, Spatial Analysis, Population Density.

\section{INTRODUÇÃO}

O processo de urbanização ocorre de maneira intensa no Brasil e em outras regiões do mundo. Entretanto, na maioria das vezes, esse crescimento ocorre de maneira desordenada, o que dificulta o planejamento e a administração de municípios. O processo de ocupação urbana pode atingir, não somente municípios, mas também suas regiões adjacentes, o que leva à formação das chamadas Regiões Urbanas Homogêneas (RUHs). Existe um problema, no entanto, em relação à definição dessas RUHs pois na maioria dos casos tais definições são baseadas em interesses políticos e administrativos, sem apresentar um critério quantitativo ou padronizado. O objetivo desta pesquisa foi a identificação e delimitação de RUHs no Brasil a partir da análise da distribuição populacional, empregando-se ferramentas de estatística espacial.

\section{REVISÃO BIBLIOGRÁFICA}

Recentes estudos das Nações Unidas (UN, 2015) mostraram que a população urbana mundial vem crescendo nos últimos anos e que continuará nesse sentido, de acordo com uma projeção feita para 2050. Como consequência, espera-se maiores níveis de relacionamento entre municípios. Uma maneira bastante coerente e objetiva de abordar o grau de urbanização dessas áreas seria adotar medidas de distribuição populacional, como a densidade populacional (UN, 2007).

No Brasil existe um grande número de municípios que estão inseridos em Regiões Metropolitanas Oficiais e, embora a instalação de RUHs esteja prevista na Constituição Federal de 1998, não existem critérios objetivos e/ou padronizados nacionalmente para que um município seja elegível ou não para fazer parte de uma região metropolitana. É de responsabilidade de cada estado a definição das RUHs, o que na maioria das vezes é feita de acordo com interesses político-administrativos (MOURA E CARVALHO, 2012).

Existe, portanto, uma necessidade de criar modelos que possam auxiliar na definição das RUHs. Breitung (2011) sugere cinco aspectos inter-relacionados que poderiam auxiliar na análise dessa delimitação: abordagens política, física, socioespacial, psicológica e funcional. Contudo, de acordo com Kourtit et al. (2015), além da dificuldade de mensuração, essas questões podem apresentar resultados ambíguos ou imprecisos.

Buscando métodos com uma abordagem mais quantitativa e de maior precisão, vários aspectos foram analisados utilizando análises espaciais, como o número de habitantes e viagens pendulares intermunicipais (OFFICE MANAGEMENT AND BUDGET, 2000), a distribuição de empregos e a oferta de infraestrutura de transportes (COOMBES, 2004) e também a densidade populacional (SANTOS, MANZATO E RODRIGUES DA SILVA, 2014). Vários autores testaram a viabilidade do uso desses indicadores, entre outros, para a definição de RUHs. Isso foi explorado por Manzato e Rodrigues da Silva (2006, 2007 e 2010); Pereira e Rodrigues da Silva (2010); Ajauskas et al. (2012); Rodrigues da Silva et al. (2014); Matiolli et al. (2016); e Aguiar, Manzato e Rodrigues da Silva (2017).

Tais estudos apontaram a técnica de estatística espacial como uma abordagem promissora para a definição e monitoramento das RUHs, considerando que as variáveis utilizadas são facilmente obtidas e manipuladas. No entanto, uma análise para todo o território nacional ainda não havia sido desenvolvida. Nesse sentido, pareceu válido identificar as RUHs brasileiras segundo a distribuição populacional. Dessa forma, seria possível apontar regiões em que a instalação de governos metropolitanos talvez não se justificasse inicialmente, além de indicar regiões que apresentam dinâmica metropolitana mas que não são reconhecidas oficialmente, fornecendo novos subsídios à questão da definição das RUHs no Brasil. 


\section{MATERIAL E MÉTODO}

A metodologia desta pesquisa envolve as bases geográficas dos municípios do Brasil e a análise dos dados sobre densidade populacional, obtidos a partir do Censo demográfico de 2010 realizado pelo IBGE (Instituto Brasileiro de Geografia e Estatística). A técnica empregada foi a análise exploratória de dados espaciais (ESDA, do inglês, Exploratory Spatial Data Analysis), um ramo da estatística espacial fundamentada na caracterização da dependência espacial, indicando como valores estão correlacionados no espaço. Este tipo de análise pode ser feita em dois tipos de dados espaciais: dados contínuos no espaço (geostatistical data) ou dados agrupados em áreas (lattice data-CRESSIE, 1993).

A técnica de ESDA apresenta três elementos básicos: uma matriz de proximidade espacial (W), um vetor de desvios (Z) e um vetor de médias ponderadas (Wz). Para a obtenção do vetor $Z$, calcula-se a média global de uma variável para todos os objetos em análise e, em seguida, subtrai-se esse valor médio encontrado do valor apresentado por cada objeto. Assim, para cada objeto é encontrado um desvio, e a união desses desvios é colocada no vetor Z. Já para o vetor Wz, calcula-se a diferença entre a média dos desvios nos objetos vizinhos e a média global. O cálculo se dá por meio da multiplicação da matriz de proximidade espacial (W) com linhas normalizadas pelo vetor transposto de desvios (expoente †).

O índice de Moran (I) é a estatística mais difundida e fornece uma medida geral da associação espacial existente em um conjunto de dados. Para valores normalizados, o índice varia de $-1 a+1$. Valores próximos de zero indicam a inexistência de autocorrelação espacial significativa entre os valores dos objetos e seus vizinhos. Valores positivos indicam autocorrelação positiva, ou seja, o valor do atributo de um objeto tende a ser semelhante aos valores dos seus vizinhos. Valores negativos para o índice, por sua vez, indicam autocorrelação negativa. O índice de Moran é calculado através da Equação (1) a seguir.

$I=\frac{Z^{t} \cdot W_{Z}}{Z^{t} \cdot Z}$

Os resultados podem ser representados em um gráfico de espalhamento de Moran, construído a partir dos valores normalizados de $Z$ por Wz, sendo esse gráfico dividido em quatro quadrantes, Q1, Q2, Q3 e Q4, e em mapas temáticos de espalhamento de Moran (Box Maps).

No quadrante Q1, também denominado HH (de High-High), o valor da variável do objeto e a média de seus vizinhos são superiores à média global. No quadrante Q2, por sua vez, chamado de LL (de Low-Low), esses valores são inferiores à média global. Dessa forma, nesses dois quadrantes, visualiza-se uma autocorrelação espacial positiva. Já os quadrantes Q3 e Q4 apresentam uma autocorrelação espacial negativa, tendo em vista que o objeto em análise difere de seus vizinhos em relação ao caráter estudado. No quadrante Q3, ou LH (de LowHigh), o valor do atributo do objeto é inferior à média global e a média dos atributos adjacentes é superior à essa média, enquanto no quadrante Q4, ou HL (de High- Low), ocorre o contrário.

Para a manipulação dos dados, aplicação da técnica e análise dos resultados, foi utilizado o software de Sistemas de Informação Geográfica (SIG) de distribuição livre denominado Terraview, o qual é desenvolvido pelo Instituto Nacional de Pesquisas Espaciais (INPE), na versão 4.2.2.

\section{RESULTADOS}

A análise espacial foi feita, primeiramente, gerando uma matriz de proximidade baseada nos valores de densidade populacional de cada município, obtendo um Índice Moran para cada estado. Com exceção do estado de Roraima, todos os estados analisados apresentaram valor positivo para índice de Moran, o que significa que os municípios apresentaram uma autocorrelação espacial positiva, mostrando existir uma dependência espacial entre eles (Tabela 1). 
Para a distribuição dos municípios nos quatro quadrantes de Moran, o valor médio para comparação e obtenção dos vetores $Z$ e Wz foi o da densidade populacional de cada estado. Assim, cada município foi alocado de acordo com a comparação da sua densidade populacional, e da média dos seus vizinhos, com a média da densidade populacional do estado no qual está inserido.

Tabela 1 - Índice de Moran dos Estados brasileiros

\begin{tabular}{|c|c|c|}
\hline Região & Estado & Índice de Moran \\
\hline \multirow{7}{*}{ Norte } & $A C$ & 0,029 \\
\hline & $A M$ & 0,048 \\
\hline & AP & 0,122 \\
\hline & PA & 0,354 \\
\hline & $\mathrm{RO}$ & 0,213 \\
\hline & RR & $-0,135$ \\
\hline & TO & 0,192 \\
\hline \multirow{9}{*}{ Nordeste } & $\mathrm{AL}$ & 0,131 \\
\hline & BA & 0,164 \\
\hline & CE & 0,156 \\
\hline & MA & 0,239 \\
\hline & PB & 0,507 \\
\hline & PE & 0,593 \\
\hline & $\mathrm{Pl}$ & 0,112 \\
\hline & RN & 0,197 \\
\hline & SE & 0,140 \\
\hline \multirow{3}{*}{ Centro Oeste } & $G O$ e DF & 0,180 \\
\hline & MS & 0,085 \\
\hline & MT & 0,201 \\
\hline \multirow{4}{*}{ Sudeste } & ES & 0,297 \\
\hline & $M G$ & 0,394 \\
\hline & RJ & 0,577 \\
\hline & SP & 0,533 \\
\hline \multirow{3}{*}{ Sul } & PR & 0,332 \\
\hline & RS & 0,640 \\
\hline & SC & 0,286 \\
\hline
\end{tabular}

Fonte: Os autores

Os resultados obtidos foram representados com cores diferentes para cada quadrante. Observa-se que, a partir da técnica de análise espacial ESDA, as regiões que apresentam dinâmica metropolitana são obtidas a partir de agrupamentos de municípios classificados como HH circundados por municípios LH (Figura 1). Aqueles classificados como HL estão usualmente dispersos na área total considerada, mas também podem situar-se próximo de municípios $\mathrm{HH}$ e LH. Entretanto, observa-se que alguns municípios classificados como $\mathrm{HH}$ e $\mathrm{LH}$ também aparecem dispersos na região analisada.

Destacando-se, por exemplo, o estado de São Paulo (Figura 2), os agrupamentos de municípios identificados como HH circundados por municípios LH ficam bastante evidentes, 
além daqueles classificados como HL dispersos pelo estado.

As Regiões Urbanas Homogêneas são identificadas pelos agrupamentos de municípios $\mathrm{HH}$ e LH, que de acordo com a técnica são as regiões que apresentam uma dinâmica metropolitana. Observam-se, também, as semelhanças e divergências entre essas regiões e as regiões delimitadas pelo traço mais espesso, que representa as regiões metropolitanas oficiais brasileiras (Figuras 3 e 4).

Figura 1 - Resultados ESDA e contorno das Regiões Metropolitanas Oficiais no Brasil

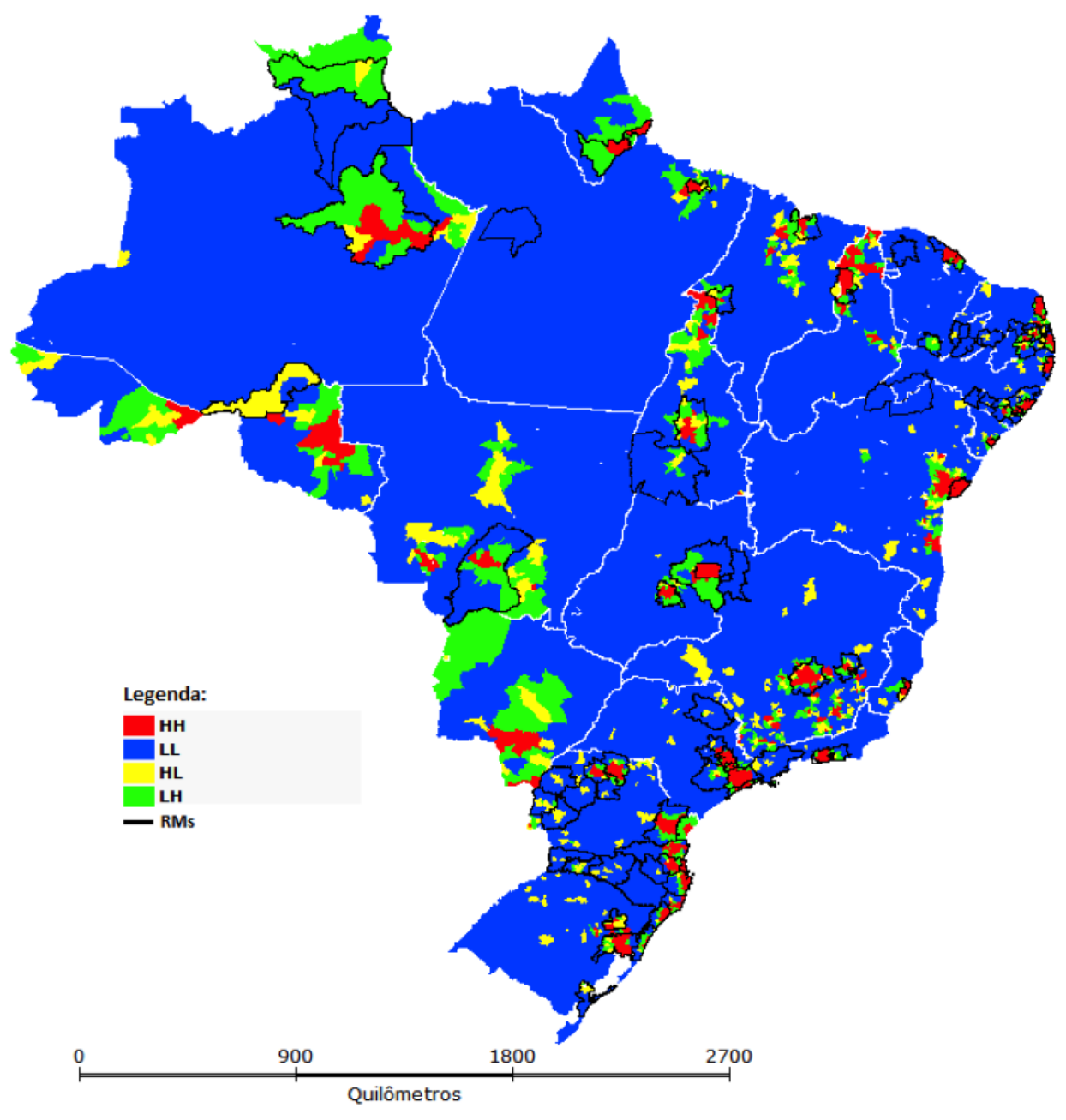

Fonte: Os autores 
Figura 2 - Resultados ESDA e contorno das Regiões Metropolitanas Oficiais no Estado de São Paulo

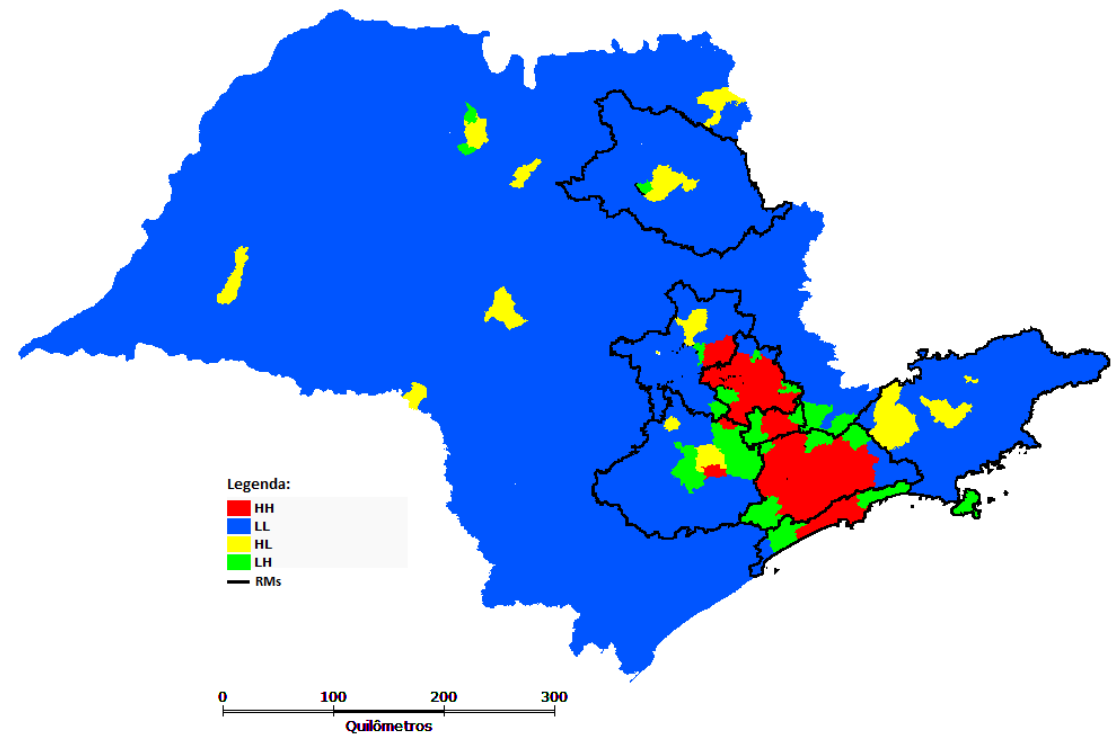

Fonte: Os autores

Figura 3 - RUHs definidas pelo método e contorno das Regiões Metropolitanas Oficiais no Brasil

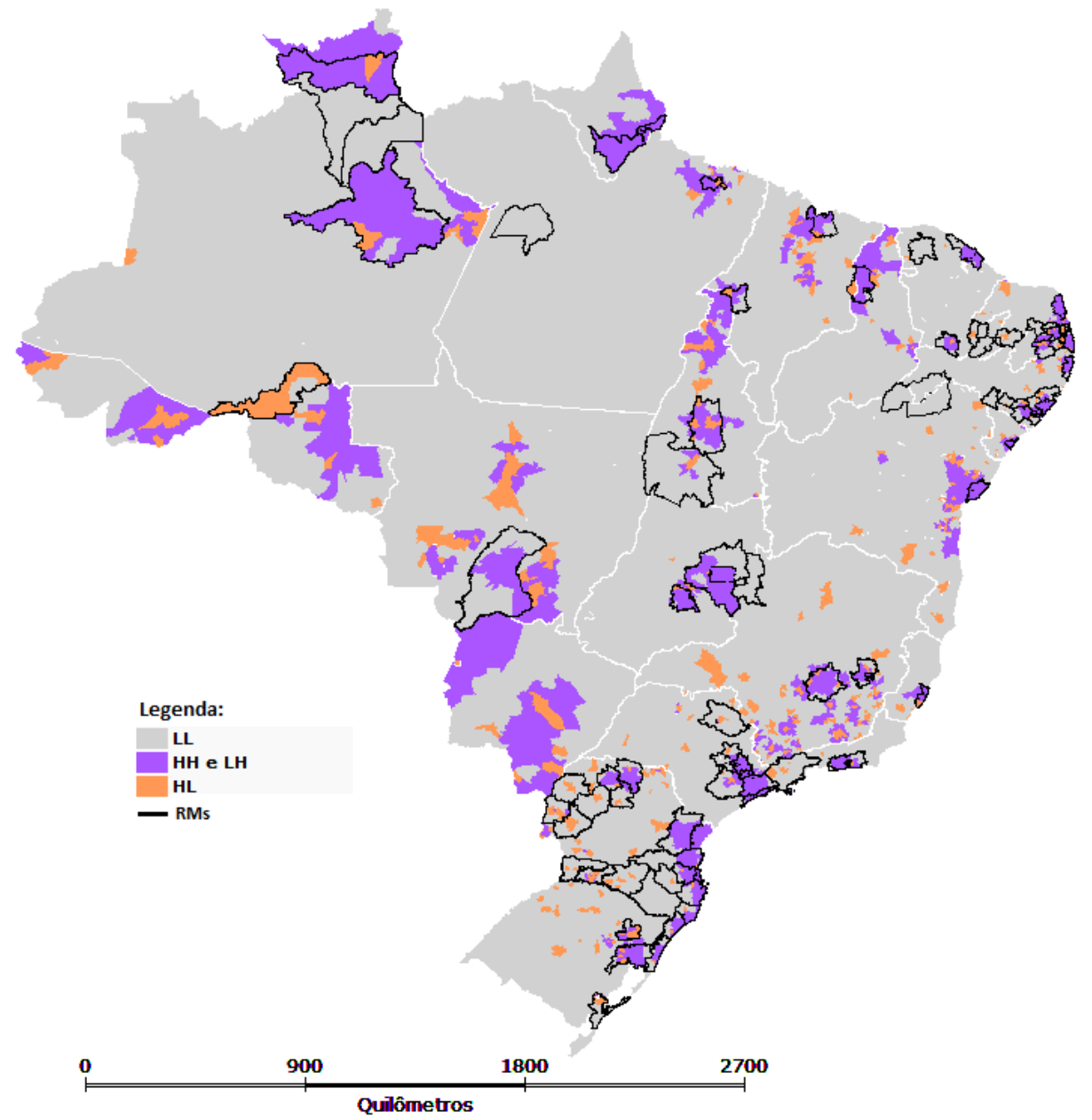

Fonte: Os autores 
Figura 4 - RUHs definidas pelo método e contorno das Regiões Metropolitanas Oficiais no Estado de São Paulo

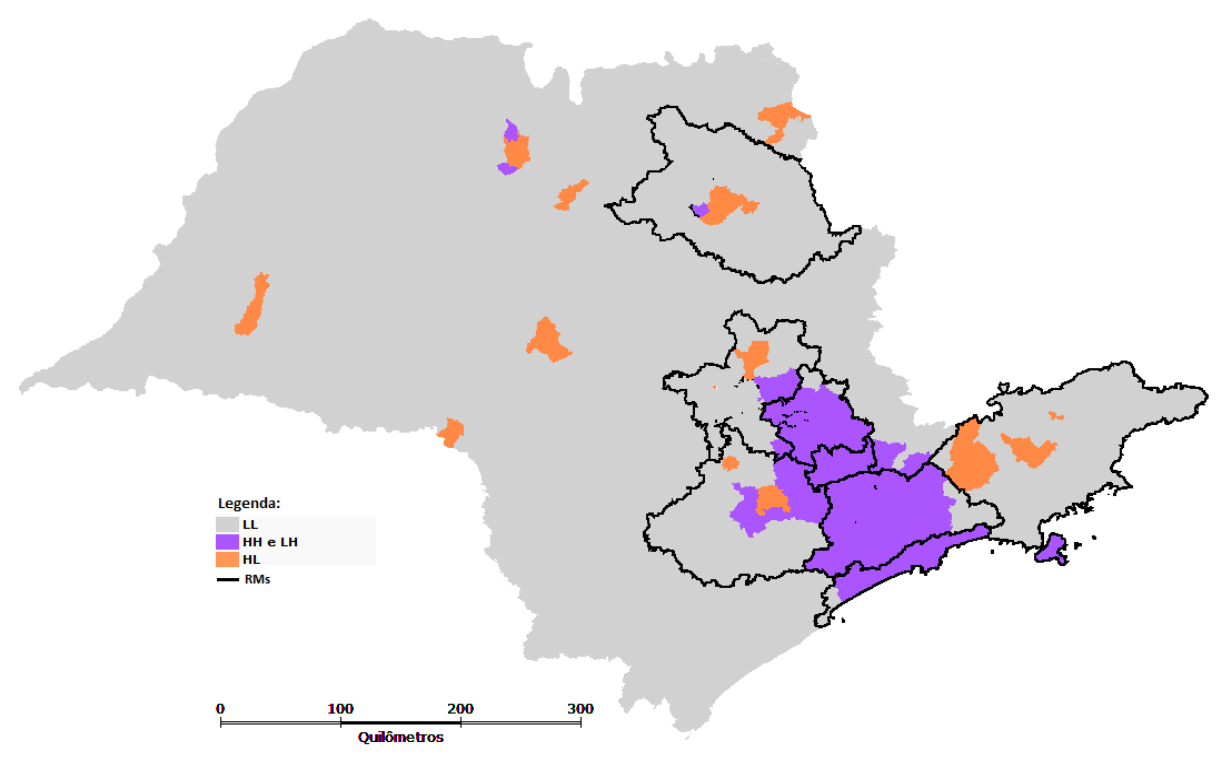

Fonte: Os autores

Observa-se que existem coincidências significativas entre as Regiões Metropolitanas Oficiais e as RUHs delimitadas pelo método, como por exemplo a Regiões Metropolitanas de São Paulo e de Manaus. Entretanto, ocorreram também divergências, tanto da existência de regiões oficiais que não foram identificadas pelo método, quanto de regiões delimitadas pela técnica ESDA e que não são consideradas com dinâmica metropolitana oficialmente, como nos estados do Pará e do Mato Grosso do Sul, respectivamente.

Considerando o somatório de municípios pertencentes a Regiões Metropolitanas Oficiais como Conjunto A, com 1379 elementos; somatório de municípios ESDA HH como Conjunto B e somatório de municípios ESDA LH como Conjunto C, totalizando B+C com 1035 elementos; obteve-se a intersecção dos conjuntos $A \cap[B+C]$ resultando em 559 municípios, ou seja, 559 municípios do Brasil fazem parte, tanto de Regiões Metropolitanas Oficiais, quanto das RUHs delimitadas por esse estudo.

\section{CONCLUSÕES}

O método apresentou resultados coincidentes para um número significativo de municípios que estão inseridos, tanto nas RUHs geradas a partir da técnica ESDA, quanto nas regiões metropolitanas oficiais. Entretanto, foi observado que as regiões oficiais não apresentaram total concordância com as regiões produzidas pela técnica, resultando em divergências entre municípios inseridos nessas regiões e aqueles caracterizados como pertencentes a RUHs pela técnica de análise baseada na distribuição populacional.

A técnica ESDA apresentou um bom desempenho na definição das RUHs quando utilizado o parâmetro de densidade populacional dos municípios. O estudo mostrou que a análise aqui proposta é bastante válida e efetiva, principalmente por se basear em referências quantitativas. O método leva a uma definição mais objetiva, possível de ser utilizada na identificação das RUHs, abrindo ainda a possibilidade de ser combinada com outras variáveis relevantes, e que poderia produzir um método de delimitação padronizado.

\section{REFERÊNCIAS}

AGUIAR, L. L.; G. G. MANZATO e A. N. RODRIGUES DA SILVA (2017) Patterns of commuting flows for delimitating Functional Urban Regions in the state of São Paulo, Brazil. Proceedings of 
the 15th International Conference on Computers in Urban Planning and Urban Management (CUPUM), Adelaide, Australia.

AJAUSKAS, R.; MANZATO, G. G. e RODRIGUES DA SILVA, A. N. (2012) The Definition of Functional Urban Regions: Validation of a Set of Spatial Models with Recent Census Data and Analysis of an Additional Model Specification. Proceedings of CAMUSS, the International

Symposium on Cellular Automata Modeling for Urban Spatial Systems, Porto, Portugal.

BREITUNG, W. (2011) Borders and the City: Intra-Urban Boundaries in Guangzhou (China). Quaestiones Geographicae, Vol. 30, No. 4, 55-61.

COOMBES, P. P. e OVERMAN, H. G. (2004) The spatial distribution of economic activities in the European Union. In J. V. Henderson e J. F. Thisse (eds.), Handbook of Urban and Regional Economics, Vol. 4, Cities and Geography, North Holland, Amsterdam, 2845-2909.

CRESSIE, N. (1993) Statistics for Spatial Data. Wiley, New York.

Dias, R. S., Manzato, G. G. e Rodrigues da Silva, A. N. (2014). A capacidade de infraestrutura rodoviária e sua relação com o processo de metropolização. XXVIII Congresso de Pesquisa e Ensino em Transportes, Curitiba, Brasil.

KOURTIT, K.; P. NIJKAMP e M. D. PARTRIDGE (2015) Challenges of the New Urban World. Applied Spatial Analysis and Policy, Vol. 8, No. 3, 199-215.

MANZATO, G. G. e RODRIGUES DA SILVA, A. N. (2006) Incorporando um indicador de oferta de infraestrutura de transportes na definição de regiões metropolitanas. $\mathbf{X} \mathbf{X}$ Congresso de Pesquisa e Ensino em Transportes, Brasília, Brasil, Vol. 1. 341-352.

MANZATO, G. G. e RODRIGUES DA SILVA, A. N. (2007) Uma estrutura conceitual para a definição de regiões urbanas homogêneas. XXI Congresso de Pesquisa e Ensino em Transportes, Rio de Janeiro, Brasil.

MANZATO, G. G. e RODRIGUES DA SILVA, A. N. (2010) Spatial-temporal combination of variables for monitoring changes in metropolitan areas, Applied Spatial Analysis and Policy, Vol. 3, No. 1, 25-44.

MATIOLLI, J. A. C., OLIVEIRA JUNIOR, M. A. e MANZATO, G. G. (2016) Modelagem espacial para a definição de Regiões Urbanas Homogêneas incorporando recentes dados demográficos e de oferta de infraestrutura rodoviária. XXX Congresso de Pesquisa e Ensino em Transportes, Rio de Janeiro, Brasil.

MOURA, R. e CARVALHO, I. (2012) Estatuto da Metrópole: onde está a região metropolitana? Observatório das Metrópoles, Disponível em:

http://observatoriodasmetropoles.net/index.php?option=com_k2\&view=item\&id=455\%3Aest atuto-da-metr\%C3\%B3pole-onde-est\%C3\%A1-a-

regi\%C3\%A3ometropolitana\%3F\&ltemid=165\&lang=pt Acesso em 15/04/2014.

OFFICE OF MANAGEMENT AND BUDGET (2000) Standards for defining metropolitan and micropolitan statistical areas, Federal Register, Vol. 65, No. 249, December 27, 2000. PEREIRA, H. T. S. e RODRIGUES DA SILVA, A. N. (2010) Comparing spatial analysis methods for the definition of Functional Urban Regions - The case of Bahia, Brazil. 10 $0^{\text {th }}$ International Conference on Design and Decision Support Systems in Architecture and Urban Planning, Eindhoven, The Netherlands.

RODRIGUES DA SILVA, A. N.; G. G. MANZATO e H. T. S. PEREIRA (2014) Defining Functional Urban Regions in Bahia, Brazil, using roadway coverage and population density variables. Journal of Transport Geography, Vol. 36, 79-88. DOI:10.1016/j.jtrangeo.2014.03.001. 
SANTOS, S. P.; G. G. MANZATO e A. N. RODRIGUES DA SILVA (2014) Estratégias para a determinação da densidade populacional visando a definição de regiões urbanas homogêneas por meio de técnicas de análise espacial. Anais do $6^{\circ}$ Congresso Luso Brasileiro para o Planejamento Urbano, Regional, Integrado e Sustentável, Lisboa, Portugal.

UN (2007) United Nations, Population fund, state of world population 2007, New York: UN.

UN (2015) United Nations, Department of Economic and Social Affairs, Population Division. World Urbanization Prospects: The 2014 Revision. 